\title{
Tratamiento de la Infertilidad Femenina de Causa Tubárica con Microcirugía
}

\author{
Dr. Jorge E. Corzo C.* - Dr. Alfonso López Jiménez*
}

\section{INTRODUCCION}

A medida que aumenta el número de esterilizaciones en la mujer aumenta también el número de pacientes que desean revertir esa esterilización. Como respuesta a esta situación se han ideado métodos quirúrgicos tendientes a restablecer su fertilidad $(2,3)$.

Uno de estos métodos es la macrocirugía; varios investigadores han trabajado en este campo, entre ellos Hellman desde $1951(17,25)$.

Otro método es la microcirugía, cuya aplicación en Ginecología es muy reciente si se compara con el uso en otros campos de la Medicina. Los primeros en usarla fueron los oftalmólogos en 1887 , en 1956 la utilizó Barraquer en Colombia (1), los otorrinolaringólogos la aplicaron en 1921, los cirujanos vasculares en 1960 (16), los neurocirujanos en 1961 y, finalmente, los ginecólogos y urólogos en 1967 (26). Swolin fue el pionero de la microcirugía y en 1967 la utilizó en el tratamiento de infertilidad femenina de causa tubárica.

Casi simultáneamente otros autores, entre ellos Gomel (15), Winston y Acland (28), en diferentes partes, estudiaron y mejoraron técnicas de micro-

* Especialistas Depto. de Gineco-Obstetricia Hospital Militar Central, Bogotá, noviembre de 1981. cirugía para restablecer la fertilidad en pacientes previamente esterilizadas, así como para restablecer la anatomía y fisiología de trompas alteradas por procesos inflamatorios $(13,23,29)$.

Entre nosotros se han aprovechado las experiencias de los autores mencionados anteriormente en el campo de la ginecología; el trabajo que presentamos es el informe preliminar de un esfuerzo orientado a resolver los problemas de in-fertilidad de causa tubárica.

\section{Propósito}

El propósito de este trabajo es restablecer la capacidad reproductora de mujeres con infertilidad de orígen tuboperitoneal, ya sea secundaria a esterilización quirúrgica por las técnicas de bandas. cauterio o Pomeroy, o secundaria a procesos inflamatorios.

Además, incluye un grupo de pacientes con infertilidad primaria de orígen tubárico.

El objetivo fue revertir la esterilización tubárica de orígen quirúrgico, utilizando la técnica de microcirugía, y eliminar el factor tuboperitoneal que interfiere con la fertilidad de las pacientes que consultaron durante el período comprendido entre marzo de 1980 y octubre de 1981. 


\section{Material y métodos}

Nos parece conveniente resumir en forma breve lo relacionado con Anatomía y Fisiología de las trompas, conceptos sobre microcirugía, causas de obstrucción tubárica.

\section{Anatomía y Fisiología de}

\section{Trompas $(4,5,8,9,21)$}

La trompa de Falopio se describe en 4 segmentos anatómicos: la porción intersticial o intramural, incluída en la pared del útero; la porción istmica, inmediatamente por fuera de la unión útero-tubárica; la porción ampular y el infundíbulo, en cual termina en la fimbria. La longitud promedio de la trompa es de $11-12 \mathrm{~cm}$.

La porción intramural sigue un curso indefinido, recto, curvo o tortuoso dentro de la pared uterina; la mucosa de la porción intramural se encuentra en transición entre la mucosa uterina y la tubárica sin que exista una línea definida de separación entre ellas. Su capa muscular es rica en fibras y es bien definida; su longitud es aproximadamente de $2.5 \mathrm{~cm}$.

La porción istmica mide $2-3 \mathrm{~cm}$, con tiene la musculatura más gruesa de toda la trompa; el diámetro de la luz es en promedio de 400 micras y la mucosa está usualmente conformada en cuatro pliegues.

La ámpula es la porción más larga de la trompa, mide $5-8 \mathrm{~cm}$,su luz varía de $4 \mathrm{~mm}$, en la unión con el istmo, hasta cerca de $1 \mathrm{~cm}$ en su parte distal; la musculatura es delgada porque tiende a colapsarse. La cirugía tubárica a este nivel es más exigente $y$ menos agradecida.

El infundíbulo es la porción distal del oviducto terminando en la fimbria y permite el transporte fisiológico del óvulo. La cirugía a este nivel implica una técnica más sofisticada.
La irrigación se deriva de las arterias uterinas y ovárica. La porción intersticial $y$ los dos tercios medios del oviducto son irrigados por la arteria tubárica interna, rama de la uterina y la porción restante por la tubárica externa, rama de la ovárica. El compromiso del arco vascu lar formado por estas arterias y su correspondiente arco venoso de retorno, podría comprometer de manera importante los resultados quirúrgicos de la trompa en cuanto a fertilidad se refiere.

La inervación, simpática y parasimpática, está dada por filetes nerviosos derivados de T10-L2 para el istmo y algunas porciones de la ámpula, y por filetes nerviosos del plexo hipogástrico para la porción distal.

El epitelio de la trompa tiene cuatro diferentes células descritas hasta el momento: ciliadas, secretoras, intercalares (en espiga) e indiferentes. Las células ciliadas se encuentran en gran número en la fimbria y ámpula y disminuyen en los segmentos restantes. Las células secretoras varían en apariencia y contenido durante el ciclo menstrual. Las células intercalares pueden representar células exhaustas o células deciliadas.

\section{Fisiología:}

1. Transporte de espermatozoides: de los millones de espermatozoides depositados en la vagina, solamente miles llegan al oviducto y sólo algunas decenas llegan al lugar de la fertilización. Se han recuperado espermatozoides del oviducto humano dentro de los treinta minutos siguientes a su colocación en la vagina.

2. Capacitación: es el desarrollo de la habilidad para fecundar, fenómeno que parece involucrar la desestabilización de la membrana plasmática que cubre la cabeza del espermatozoide. No se ha pro bado en el humano.

3. Captación del Ovulo: implica mecanismos de aposición con el ovario $v$ 
mecanismos de captación del fondo de saco; tendría que ver con mecanismos de movimientos propios de las cilias y de la musculatura de la trompa, mesosalpin $x$ y mesovario.

4. Cambios en el Ovulo: el óvulo gasta algún tiempo en el oviducto antes de ser receptivo para la penetración del espermatozoide. Parece existir una propiedad de interfase entre el óvulo y el endosalpinx para que exista un transporte eficiente del óvulo.

5. Fertilización: el fluido de la trompa provee suficiente medio ambiente para la fertilización; sin embargo, no parece ser indispensable para que ocurra la fertilización.

6. Transporte del embrión: una vez fertilizado el óvulo permanece en la trompa por varios días (5 días), siendo su estadía más larga en la ámpula y pasando rápidamente a traves del istmo.

\section{¿Qué es microcirugia? $(19,27,29,30)$}

La microcirugía no es una forma nueva de cirugía, sino un método que, además de utilizar magnificación, involucra el concepto de manejo delicado de los tejidos, hemostasis meticulosa y la utilización de suturas e instrumental finos.

\section{Principios de microcirugía $(10,11,12,14)$}

Son pasos indispensables durante el acto quirúrgico con la técnica de microcirugía; fueron establecidos en diferentes partes del mundo donde se practica este tipo de cirugía, y pueden sintetizarse en la forma siguiente:

\section{Mejorar el campo visual operatorio} con magnificación (lupa o microscopio). El microscopio es un instrumento binocular que provee un sistema de luz coaxial para iluminación óptima; el objetivo ideal para cirugía humana es de $25-30 \mathrm{~cm}$ de longitud focal para pro- veer un adecuado campo operatorio entre el lente y el paciente; la magnificación varía entre $10-20 \times$ y puede ser ajustada para compensar la visión del cirujano, aunque casi nunca se utiliza tanta magnificación para cirugía tubárica, ya que magnificaciones grandes reducen la profundidad del campo $y$, también el diámetro del campo visual.

Las lupas tienen una longitud focal que va de $30-49 \mathrm{~cm}$ y la magnificación varía entre 3 y 6 X, siendo el campo inversamente proporcional a la magnificación; la luz debe ser aumentada por cualquier sistema independiente para iluminar el campo operatorio, resultando más económica, fácil de transportar y maniobrar y reduciendo considerablemente el tiempo operatorio en relación con el microscopio.

2. Preparación del campo operatorio: a fin de lograr una buena exposición de los órganos pélvicos donde se practicará la microcirugía, se procede a colocar una cantidad variable de mechas en vagina con el fin de elevar el útero y anexos. Deberá practicarse una incisión amplia -Pfannestiel o mediana- que permita una buena exposición de los órganos pélvicos; en ocasiones es útil colocar compresas húmedas en el fondo de saco de Douglas para mejorar la elevación del útero.

3. Irrigación permanente en los tejidos: mientras la cavidad peritoneal permanece expuesta, los tejidos se someten a un resecamiento que podría causar trauma celular interfiriendo con la buena restauración posterior; para evitarlo se utilizan soluciones (solución salina, hidrocortisina y heparina) para la irrigación constante de los tejidos.

4. Hemostasis: deben identificarse exactamente los sitios de sangrado utilizando para ello la irrigación para facilitar la hemostasis la cual se hace por coagulación ya sea con electrodo bipolar o monopolar, tratando en lo posible de 
causar mínimo daño al tejido; el microelectrodo limita el daño tisular debido al tamaño pequeño de la punta; la irrigación simultánea disipa el calor disminuyendo el trauma al tejido.

5. Manejo delicado de los tejidos: el manejo delicado de los tejidos deberá comenzar desde el momento de la incisión de la laparotomía, evitando al máximo el sangrado, utilizando irrigación constante, manejando cuidadosamente la trompa y el ovario, preferiblemente con las pinzas diseñadas para tal fin; debe evitarse sujetar las trompas con pinzas de punta fina o con garra, ya que cualquier desgarro tisular o trauma por exceso de manipulación, interferirá directamente con el buen resultado de la cirugía.

No deberán quedar zonas cruentas en el campo operatorio y cuando sea necesario, se aplicarán injertos de peritoneo para cubrirlas. Deberá prestarse especial atención a la remoción de todo tejido necrótico del campo operatorio, ya que su presencia podría liberar el mecanismo de formación de adherencias.

6. Utilización de suturas e instrumental finos: se utilizan suturas no absorbibles de 8-10 ceros, ya que las suturas al absorberse producen reacción tisular que podría causar zonas extensas de fibrosis, que serán mayores en cuanto mayor sea la cantidad de sutura utilizada; por eso se recomienda utilizar estrictamente los puntos necesarios. Los instrumentos básicos requeridos son: portagujas con o sin seguro, pinzas de disección con y sin garra, microtijeras $y$ pinzas para manejo de trompas y ovarios; además se usan tubos de vidrio de varios tamaños para la movilización e intervención de trompas.

\section{Selección de pacientes y estudio preoperatorio $(10,20)$}

Se presentan 33 pacientes intervenidas durante el tiempo comprendido entre marzo de 1980 y octubre de 1981 y quienes consultaron por infertilidad.

En la primera entrevista con la pareja que deseaba reversión de la esterilización o con esterilidad primaria de origen tuboperitoneal, se les explicó el procedimiento a seguir $y$ las posibilidades de éxito en cada caso. Algunas parejas desistieron del tratamiento quirúrgico al conocer en forma realista los resultados esperados.

Antes de efectuar la reconstrucción tubárica se descartaron todos los demás factores de infertilidad. La motivación principal que hizo consultar a la pareja se muestra en el Cuadro No. 2, en donde se ve que el deseo de un nuevo embarazo dentro del mismo matrimonio fue el principal factor que intervino en la decisión de someterse a la cirugía, generalmente por mejor status económico y sentimiento de "falta de un hijo pequeño".

Los estudios preoperatorios incluyeron HSG, laparoscopia y todos aquellos que ayudan a determinar la causa de infertilidad, tales como temperatura basal, biopsia de endometrio, test de Huhner, espermograma, dosificaciones hormonales, etc.

La HSG es un procedimiento relativamente simple y está asociado con una baja rata de complicaciones; provee información sobre la cavidad uterina y la luz de las trompas. Tiene limitaciones para ver las adherencias peritubáricas.

La laparoscopia permite completar la visualización de la cavidad pélvica y en especial las trompas y su patología, además de facilitar establecer la naturaleza y distribución de las adherencias.

\section{Clasificación de la lesión}

Las pacientes se clasificaron en 3 grupos: lesión leve, moderada y severa, 
de acuerdo a la severidad de obstrucción. Para la clasificación de la lesión se tuvieron en cuenta los siguientes parámetros: presencia y tamaño del hidrosal pinx, con servación 0 no de los pliegues del endosalpin $\mathrm{x}$, presencia y tipo de adherencias (laxas o densas), presencia de tumores uterinos u ováricos, presencia o no de endometriosis, longitud y estado - de las trompas.

Lesión leve: ausencia de hidrosalpin $\mathrm{x}$ o si lo hay, que sea menor de $15 \mathrm{~mm}$, conservación de los pliegues del endosalpinx, adherencias laxas o no existentes, obstrucción de tipo quirúrgico a nivel istmico o ampular, con conservación de trompa sana igual o mayor de 6 a $8 \mathrm{~cm}$ con buena conservación de la fimbria.

Lesión moderada: hidrosalpinx de 15 a $30 \mathrm{~mm}$, conservación del patrón de los pliegues del endosalpinx, adherencias que no fijan los órganos pélvicos.

Lesión severa: hidrosalpinx mayor de $30 \mathrm{~mm}$, ausencia de rugosidades (pliegues) del endosalpinx, ausencia de fimbria, adherencias densas, vascularizadas, endometrios, tumores. La obstrucción de tipo quirúrgico asociada a adherencias densas, a procesos endometriósicos o tumorales, se considera lesión severa. La presencia de uno sólo de estos factores implica clasificación de lesión severa.

Las revisiones existentes en la literatura asignan un porcentaje de éxito que va desde el $60-75 \%$ para las lesiones leves, $45 \%$ para las moderadas y menos del $30 \%$ para las lesiones severas.

\section{Causas de obstrucción tubárica}

Las causas de oclusión tubárica podemos clasificarlas de la forma siguiente:

\section{A. Esterilización quirúrgica}

1. Con sección de un fragmento de trompa
2. Ligadura o banda.

3. Cauterización de una porción de la trompa

B. Resultado de Infecciones

1. Bloqueo cornual - salpingitis ístmica nodosa

2. Bloqueo sin distensión debido a estreptococo o estafilococo, otros.

3. Hidrosalpinx - gonococo, TBC, estreptococo o estafilococo

C. Adherencias peritubáricas causadas por:

1. salpingitis

2. apendicitis

3. endometriosis

4. cirugías pélvicas previas

D. Secundaria a tumores benignos o malignos de órganos adyacentes, (miomas uterinos, tumores de ovario, etc.).

\section{Contraindicaciones para microcirugía(10)}

Existen algunas situaciones que contraindican la cirugía tubárica reconstructora:

1. $T B C$ genital. Cuando la oclusión tubárica es debida a TBC genital, los resultados de la cirugía son extremadamente pobres aún después de completar el tratamiento médico. Aún en el caso de trompas permeables con la HSG, a la laparoscopia podemos encontrar lesiones miliares en la pelvis y observar escape de material caseoso a través de las trompas en casos totalmente insospechados.

La HSG es de gran ayuda y puede demostrar: a) formación de nichos en la luz de la trompa por penetración del medio en los pliegues del endosalpinx, b) constricción de la trompa con saculación distal en forma de pipa, c) luz tubárica con irregularidades $\mathrm{y}$ distintas 
constricciones en forma de vaina de alverja, d) trompa gruesa mostrando ventanas irregulares como botones en su parte distal, e) nódulos linfáticos calcificados, o calcificaciones en la luz de. la trompa.

2. Trompas escleróticas, duras y adherencias densas que conglutinan los órganos pélvicos.

3. Mujeres por encima de 38 años de edad, debido al gran descenso de la rata de fertilidad y al aumento de las posibilidades de problemas congénitos.

4. En presencia de enfermedad inflamatorio activa.

5. La cirugía tubárica es un procedimiento electivo y no debería efectuarse como adición a cirugías de urgencia.

\section{Técnica quirúrgica $(27,30)$}

En las intervenciones quirúrgicas practicadas hemos seguido, con pequeñas modificaciones, la técnica de microcirugía que aprendimos del Dr. John Rock del Hospital Johns Hopkins de Baltimore, que a continuación describiremos a grandes rasgos:

Previa preparación de la paciente $y$ colocación de mechas vaginales con el fin de elevar los órganos pélvicos, se efectúa de preferencia una incisión de Pfannestiel haciendo hemostasis cuidadosa. Se colocan compresas humedecidas en solución de corticoide y heparina en el fondo de saco de Douglas para mejorar el campo operatorio. Con técnica de microcirugía y amplificación con lupas, se identifican los sitios de obstrucción utilizando azul de metileno colocado a través del fondo uterino; luego se reseca la porción obstruída hasta obtener bordes sanos; le hemostasis se lleva a cabo con cauterio. A continuación se coloca un gelco de teflón No. 16 a través de la parte distal de la trompa hasta el sitio donde estaba obstruída; a través del gelco, a su vez se pasa un cateter de polietileno No. 2 el cual se introduce también a través del cabo proximal de la trompa hasta la cavidad uterina dejando allí la mayor parte del cateter. Seguidamente se sutura el meso utilizando material no absorbible 5-6 ceros, para afrontar los cabos de la trompa y para reducir la tensión en el sitio de anastomosis. Utilizando material no absorbible 7-8 ceros se realiza la anastomosis termino-terminal con tres a cuatro puntos separados interesando la capa muscular de cada uno de los cabos; la serosa se afronta en la misma forma. Finalmente se comprueba la permeabilidad con azul de metileno a través de la anastomosis hasta llegar a la fimbria.

Durante el acto quirúrgico se utilizó solución de corticoide $y$ heparina para irrigación y de esta misma solución se dejan 150 cc en cavidad pélvica. Se termina el procedimiento con el cierre de pared por planos. Cuando existe otra patología se procede según el caso: salpingostomía, salpingolisis, adhesiolisis, resección de quistes, etc. A todas las pacientes se les aplicaron $3 \mathrm{gr}$ de Cefalosporina preoperatorialmente y $3 \mathrm{gr}$ en el post-operatorio.

En las pacientes de recanalización tubárica, además de la cefalosporina, se utilizaron estrógenos en el post-operatorio a una dosis de $2.5 \mathrm{mg}$ diarios durante 20 días seguidos de $10 \mathrm{mg}$ de Provera diarios durante 5 días. Igualmente se utilizaron corticoides comenzando con$24 \mathrm{mg}$ diarios en dosis fraccionada y decrecientes hasta terminar con $5 \mathrm{mg}$ diarias vía oral.

\section{Resultados}

En el grupo de 33 pacientes de este trabajo se contabilizaron 35 casos de intervenciones con microcirugía, ya que 2 pacientes fueron reintervenidas.

La edad de las pacientes varió entre 24 y 38 años de edad, Cuadro No. 1, la paridad osciló entre 0 y 7 embarazos. 
Cuadro No. 1

\begin{tabular}{cc}
\hline Edad & No. de casos \\
\hline $24-29$ & 10 \\
$30-34$ & 14 \\
$35-38$ & 11 \\
\hline TOTAL & 35 \\
\hline
\end{tabular}

En el Cuadro No. 2 se muestra la motivación que indujo a las pacientes a solicitar la intervención quirúrgica.

Cuadro No. 2

\section{MOTIVACION PARA LA CIRUGIA TUBARICA}

\begin{tabular}{lcc}
\hline & No. de casos & $\%$ \\
\hline Deseo de nuevo hijo & 16 & $45.5 \%$ \\
Muerte de un hijo & 6 & $15 \%$ \\
Nuevo matrimonio & 5 & $14 \%$ \\
Esterilidad Primaria & 7 & $20 \%$ \\
Otras causas & 1 & $2.5 \%$ \\
\hline TOTAL & 35 \\
\hline
\end{tabular}

En el Cuadro No. 3 se indica la clasificación de las pacientes en tres grupos, dependiendo del tipo de lesión encontrada en el acto quirúrgico o en el estudio previo. Esta clasificación se hizo con el fin de observar la incidencia que tiene la suma de varios factores asociados a la esterilización quirúrgica pura sobre los resultados, comparándolos con aquellos casos en que sólo se encontró esterilización quirúrgica pura. En 17 casos se practicó anastomosis tubárica sin otro procedimiento; en 9 casos, además de la recanalización se practicó salpingolisis, salpingoplastia u ovariolisis; en 9 casos no se practicó recanalización, siendo
Cuadro No. 3

\section{CLASIFICACION DEL TRATAMIENTO SEGUN EL TIPO DE LESION}

\begin{tabular}{lccccc}
\hline Tratamiento & Leve & Moderada & Severa & Embarazo \\
\hline $\begin{array}{l}\text { Reversión de esteriliza- } \\
\text { ción sin otra patología }\end{array}$ & 15 & 1 & 1 & $8(47.6 \%)$ \\
$\begin{array}{l}\text { Reversión de esteriliza- } \\
\text { ción con salpingolisis, } \\
\text { salpingoplastia-otras }\end{array}$ & - & 4 & 5 & $1(11.1 \%)$ \\
$\begin{array}{l}\text { Factor uteroovárico y } \\
\text { peritoneal }\end{array}$ & 1 & 2 & 6 & $2(22.4 \%$ \\
\hline \multicolumn{1}{c}{ TOTAL } & & & & 11 \\
\hline \hline
\end{tabular}

cas̀os de esterilidad primaria por factor tuboperitoneal importante.

Además de esta clasificación, en el Cuadro No. 3 se muestran los resultados de embarazos logrados en los diferentes grupos, notándose una diferencia importante a favor de los casos en que solamente se practicó reversión de la esterilización.

Cuadro No. 4

\section{CLASIFICACION DE LA LESION Vs EMBARAZO}

\begin{tabular}{lccc}
\hline & Casos & Embarazo Total $\%$ \\
\hline & N A E \\
\hline Leve $\quad(16(45 \%)$ & $321654.5 \%$ \\
Moderada $7(20 \%)$ & $21-327.27 \%$ \\
Severa $12(34 \%)$ & $1--218.16 \%$ \\
\hline TOTAL 35 & 11 \\
\hline \hline
\end{tabular}

En el Cuadro No. 4 se clasifican los casos según el tipo de lesión, en leve, moderada y severa, de acuerdo con los criterios enunciados previamente. Se aprecia en este cuadro un porcentaje significativamente mayor de las pacientes 
con lesión leve; además se observa que un gran número de pacientes tenía una lesión severa, datos que se correlacionan con los resultados obtenidos. En igual forma, como era de esperarse, las pacientes con lesión leve tienen un índice mayor de embarazo, mientras que el porcentaje de embarazo en las pacientes con lesión severa, es significativamente menor.

Cuadro No. 5 NIVEL DE LA RECANALIZACION

\begin{tabular}{|c|c|c|c|c|}
\hline \multirow[b]{2}{*}{ ANASTOMOSIS } & & \multicolumn{3}{|c|}{ TIPO DE LESION } \\
\hline & & Casos & L M S & Prótesis \\
\hline \multirow{3}{*}{$\begin{array}{c}\text { Istmico-cornual } \\
1,2\end{array}$} & Bilateral & - & --- & - \\
\hline & Unilateral & 2 & $1-1$ & - \\
\hline & Bilateral & 6 & $6--$ & 3 \\
\hline \multirow[t]{2}{*}{$\begin{array}{c}\text { Istmico-Istmico } \\
2,3,4\end{array}$} & Unilateral & 6 & 123 & 5 \\
\hline & Bilateral & 8 & $71-$ & 5 \\
\hline \multirow[t]{2}{*}{$\begin{array}{l}\text { Istmico-Ampular } \\
4\end{array}$} & Unilateral & 4 & 211 & 3 \\
\hline & Bilateral & - & --- & - \\
\hline \multirow[t]{2}{*}{ Ampulo-Cornual } & Unilateral & 3 & 111 & 2 \\
\hline & Bilateral & - & --- & - \\
\hline Ampulo-Ampular & Unilateral & 1 & $-1-$ & - \\
\hline
\end{tabular}

En el Cuadro No. 5 se presenta el nivel al cual se efectuaron las diversas recanalizaciones en los 26 casos sobre 24 pacientes, Se clasifica el nivel de la anastomosis indicando si se practicó uni o bilateral y si en la misma paciente se practicó la anastomosis a dos niveles diferentes. Se encontró un mayor número de anastomosis a nivel istmico-istmico e istmico-ampular. En igual forma se muestran los casos en los cuales se utilizó cateter endotubárico como guía para efectuar la anastomosis, ya que en algunos casos dicha prótesis no se utilizó.

\section{Comentarios}

Podemos observar que la motivación que llevó a nuestras pacientes a solicitar la reversión de la esterilización varía en relación con las series informadas de otros sitios, con medio sociocultural diferente, en las cuales el nuevo matrimonio es una de las causas más importantes. En nuestra serie encontramos que el factor más sobresaliente fue el deseo de un nuevo hijo, ya fuera por mejoría de su status económico o por un factor sentimental importante. La infertilidad primaria, como era de esperarse, siguió en orden de importancia entre las causas de cirugía.

Cuando se practicó la reversión de esterilización quirúrgica, se observó que el nivel de ligadura en la mayoría de las pacientes fue istmico y ampular, lo que facilitó la técnica de anastomosis por las características del tejido a este nivel; este es un detalle de tener en cuenta en el momento de efectuar la ligadura de trompas en pacientes que tuvieren opción potencial de revertir la esterilización $(6,7,24)$, ya que en ocasiones aunque la paciente y el cirujano consideran como improbable la reversión, hemos visto casos de pacientes con gran paridad que cambian de opinión en cuanto a planificación definitiva, así no hayan sucedido calamidades en su familia ni hayan ocurrido cambios en su estado conyugal.

Las pacientes a quienes se practicó recanalización tubárica sin que se hiciera necesario el tratamiento de ningún otro factor tuboperitoneal, ovárico o uterino, fueron clasificadas como leves y es notoria la diferencia en el porcentaje de embarazos en relación con otras pacientes de esta serie clasificadas de manera diferente, lo cual está de acuerdo con los datos de otras series, en las cuales, mientras más severa es la lesión, menor es el porcentaje de embarazoEn nuestra serie tenemos un porcentaje de pacientes 
clasificadas como lesión severa, de $34.5 \%$, que se operaron debido al deseo de las pacientes a pesar de habérseles explicado las escasas posibilidades de embarazo después del tratamiento quirúrgico. La mayoría de las pacientes no llevan aún un año de seguimiento lo que limita en cierta forma el porcentaje comparativo con otras series.

Se han presentado algunos abortos, posiblemente causados por cuerpo lúteo insuficiente (6), que algunos investigadores están tratando de demostrar, como consecuencia de la interferencia que podría existir en la vascularización ovárica y su drenaje, por intersección en el arco vascular de la trompa sometida primero a ligadura y sección y posteriormente a recanalización; la tasa de producción de progesterona y su liberación en la circulación general podría verse disminuida, causando un cuerpo lúteo insuficiente para mantener un embarazo joven; basados en esta hipótesis debemos hacer hincapié en la conveniencia de dar un trato cuidadoso a la vascularización de la trompa y del ovario, conservando la mayor cantidad de ella en las esterilizaciones de tipo quirúrgico.
La utilización de cateteres endotubáricos durante la recanalización ha sido ampliamente discutida en cuanto a su conveniencia o no (18); hemos visto que durante el acto quirúrgico es de gran ayuda para lograr un mejor afrontamiento de los cabos de la trompa, pero no podríamos concluir sobre su benevolencia o perjuicio al dejarlos por varias semanas en el postoperatorio, como hecho en muchos casos.

No hemos tenido ninguna complicación quirúrgica ni postquirúrgica hasta el momento; pensamos que la utilización de antibióticos en forma profiláctica es muy valiosa para este tipo de pacientes.

En todas las cirugías se utilizó irrigación constante con una solución de corticoide y heparina, dejando al final 150 cc aproximadamente en cavidad pélvica (23). Hemos tenido la oportunidad de efectuar una segunda visión quirúrgica a 4 de estas pacientes (cesárea, ectópico y una laparoscopia), encontrando que en 3 no existía ningún tipo de adherencias y en la otra existían adherencias leves; aunque esto no permite una conclusión definitiva, pensamos que la utilización de estas mezclas durante el acto operatorio son convenientes.

\section{MICROSURGERY TREATMENT OF FEMALES UNFERTILITY CAUSED IN THE TUBES}

\section{Summary:}

35 interventions using the microsurgery technique are shown to treat female unfertility caused in the tubes.

Three groups were formed according to the degree of obstruction: SLIGHT. MODERATE and SEVERE injury. The pregnancy results for each group, that is, with slight injury $(54.5 \%)$, with moderate injury $(27.27 \%)$ and with severe injury $(18.16 \%)$ are shown.
Although this is only a preliminary work since most of the surgeries were made within the past six moths, we can conclude that the microsurgery technique used in Ginecology is of great help in solving unfertility problems caused in the tubes.

\section{Resumen}

Se presentan 35 intervenciones con la técnica de microcirugía para el tratamiento de infertilidad femenina de origen tubárico. 
Se establecieron tres grupos de acuerdo con el grado de obstrucción: con lesión LEVE, con lesión MODERADA y con lesión SEVERA. Se muestran los resultados del embarazo para el grupo con lesión leve $(54.5 \%)$ con lesión moderada $(27.27 \%)$ con lesión severa $(18.16 \%)$.

A pesar de que este trabajo es preliminar, pues muchas cirugías tienen menos de seis meses de practicadas, podemos concluir que la técnica de microcirugía aplicada en Ginecología es de mucha

\section{Bibliografía}

1. BARRAQUER JI. The microscope in ocular surgery. Am J Ophthalmol 42: 916, 1956.

2. COGNAT, MA. and DESSAPT B. The history of microsurgery. In Phillips, JM. ed. Microsurgery in gynecology. (Proceedings of the Workshop for Laparoscopy and Microsurgical Repair of the Fallopian Tube and I st International Congress of Gynecologic Microsurgery, University of California, Irvine, 1977) Downey, California, American Association of Gynecologic Laparoscopists, 1977, p. 5-8.

3. DIAMOND E. Microsurgery in infertility: instrumentation and technique. In: Phillips, JM. ed. Microsurgery in gynecology. (Proceedings of the Workshop for Laparoscopy and Microsurgical Repair of the Fallopian Tube and the Ist International Congress of Gynecologic Microsurgery, University of California, Irvine, 1977) Downey, California, American Association of Gynecologic Laparoscopists, 1977. p. 20-30.

4. DIAMOND E. A microsurgical study of the blood supply to the uterine tube and ovary. In: Phillips, JM. ed. Microsurgery in Gynecology. (Proceedings of the Workshop for Laparoscopy and Microsurgical Repair of the Fallopian Tube and the ist International Congress of Gynecologic Microsurgery, University of Califor- utilidad para resolver problemas de infertilidad de orígen tubárico.

\section{Agradecimientos}

Queremos agradecer a la John Hopkins University (Baltimore, USA), a la División Materno Infantil del Ministerio de Salud de Colombia, y en forma especial al Dr. John Rock del Hospital John Hopkins (Baltimore), por su respaldo y asistencia técnica, lo cual facilitó la realización de este trabajo.

nia, Irvine) Downey, California, American Association of Gynecologic Laparoscopists, 1977, p. 69-76.

5. DIAMOND E. Fallopian Tube Circulation as it Relates to Microsurgery, In: Phillips, JM. ed. Microsurgery in gynecology. (Proceedings of the Workshop for Laparoscopy and Microsurgical Repair of the, Fallopian Tube and the Ist International Congress of Gynecologic Microsurgery, University of California, Irvine, 1977) Downey, California, American Association of Gynecologic Laparoscopists, 1977, p. 51-62.

6. DONNEZ J., Wauters M., and Thomas K. Luteal Function After Sterilization. Obsett Gynecol 1981 Jan: 57 (1): 65-68.

7 EDDY CA, and ARCHER DA. Tubal electrophysiology after microsurgical anastomosis. In: Phillips JM. ed. Endoscopy in gynecology. (Proceedings of the 3rd International Congress on Gynecologic Endoscopy, San Francisco, California, 1977) Downey, California, American Association of Gynecologic Laparoscopists 1978. p. 428-431.

8. EDDY, CA., PhD. The use of Microsurgery in the Study of tubal Physiology, In: Phillips, JM. ed. Microsurgery in gynecology. (Proceedings of the Workshop for Laparoscopy and Microsurgical Repair of the Fallopian Tube and the ist In- 
ternational Congress of Gynecologic Microsurgery, University of California, Irvine, 1977) Downey, California, American Association of Gynecologic Laparoscopists, 1977 , p. 46-50.

9. EDDY. CA. and PAUERSTEIN, CJ., Tubal Reproductive Function and the Development of Reversible Sterilization Techniques, In: Sciarra, JJ., Zatuchni, GI., and Speidel, JJ., eds. Reversal of Sterilization, (Proceedings of a Workshop on Reversal of Sterilization, San Francisco, California) Hagerstown, Maryland, Harper and Row, 1978 (PARFR Series on Fertility Regulation) p. 100-116.

10. GOMEL V. Principles of Microsurgery for Infertility, In: Phillips, JM. ed. Microsurgery in gynecology. (Proceedings of the Workshop for Laparoscopy and Microsurgical Repair of the Fallopian Tube and the Ist International Congress of Gynecologic Microsurgery, University of California, Irvine, 1977) Downey, California American Association of Gynecologic Laparoscopists, 1977. p. 114118.

11. GOMEL V. and McCOMB P. Microsurgery in gynecology. In: Silber SJ. ed. Microsurgery. Baltimore, Maryland, Willians and Wilkins 1979. p. 143-183.

12. GRUNERT, GM., DRAKE, TS., TAKA$\mathrm{KI}$, NK. Microsurgical Reanastomosis of the Fallopian Tubes for Reversal of Sterilization Obstet Gynecol 58: 148,1981

13. GARCIA, CR., MASTROIANNI, LM. Microsurgery for treatment of Adnexal Disease, Fertil Steril Vol. 34 No. 5, Nov. 1980.

14. GOMEL V. Tubal Reanastomosis by Microsurgery, Fertil Steril 28, 59-65, 1977.

15. GOMEL V. Microsurgery for Reversal of Female Sterilization, In: Sciarra, JJ., Zatuchni, GI. and Speidel JJ., eds, Reversal of Sterilization, (Proceedings of a Workshop on Reversal of Sterilization,
San Francisco, California) Hagerstown, Maryland, Harper and Row, 1978. (PARFR Series on Fertility Regulation) $p$. 195-206.

16. JACOBSON, JH., SUAREZ EO, Microsurgery in anastomosis of small vessels, Surg Forum 9: 243, 1960.

17. LOPEZ LA y CORZO JE. Reversión de Esterilización Tubárica por la Técnica de Macrocirugía, Rev. Col. Obst y Ginec. Vol. 5, p. 323-328.

18. MELDRUM DR. Microsurgical Tubal Reanastomosis. The Role of Splints, Obst. Gynecol 57: 613, 1981.

19. PHILLIPS, JM. Introduction to Gynecologic Microsurgery, In: PHILLIPS, JM. ed. Microsurgery in Gynecology, (Proceedings of the Workshop for Laparoscopy and Microsurgical Repair of the Fallopian Tube, University of California, Irvine, 1977) Downey California, American Association of Gynecologic Laparoscopists 1977, p. 2-4.

20. QUINLIVAN, WLG., The Selection of Patients for Tubal Surgery, In: Phillips JM. ed. Microsurgery in Gynecology, (Proceedings of the Workshop for Laparoscopy and Microsurgical Repair of the Fallopian Tube, University of California, Irvine, 1977) Downey, California, American Association of Gynecologic Laparoscopists, 1977 p. 105-108.

21. Sweeney, WJ., The interstitial portion of the uterine tube: its gross anatomy, course and length. Obstetrics and Gynecology 19: 3-8, 1962.

22 SWOLIN K., Fertilitatsoperationen. 1. Literatur und Methodik, Acta Obstet, Gynecol Scand 46: 234, 1967.

23. SWOLIN K., Experimental studies on the Prophylaxis of Post-operative intra-abdominal adhesions, Studies of lipid and prednisolone Acta Obstet Gynecol Scand 45: 473, 1966. 
24 SEILER, JS. et al., Tubal Sterilization by Bipolar Laparoscopy: Report of 232 Cases, Obstet Gynecol 58: 92, 1981.

25. SIEGLER, AM. and Hellman, LM., Tubal Plastic Surgery, Fertil Steril 7 (2), 170177, March-April 1956.

26. SILBER SJ., Microsurgery in clinical urology, Urology 6: 150, 1975.

27. SIEGLER, AM. and Kontopoulos V. Reversal of tubal sterilization: implications, techniques and results. In: Sciarra, JJ., Zatuchni, JJ., eds. Reversal of Sterilization, (Proceedings of a Workshop on Reversal of Sterilization, San Francisco,
California) Hagerstown, Maryland, Harper and Row, 1978 (PARFR Series on Fertility Regulation) p. 134-142.

28. WINSTON, RL., M.R.C.O.G.: Microsurgery of the Fallopian Tube; From Fantasy to Reality, Fertil Steril Vol. 34 No. 6, December 1980, 521-530.

29. WALLACH, EE., Tubal Reconstructive Surgery 1980, Fertil Steril Vol. 34 No. 6 December 1980, 531-533.

30. WINSTON RML., Microsurgical tubocor nual anastomosis for reversal of Sterilization. Lancet 1: 284, 1977. 